\title{
Synthesis, Characterizations and Applications of Tricalcium Phosphate as A Bone Substitute
}

\author{
Alexandre Cestari* \\ Federal Institute of Education, Science and Technology of São Paulo, Brazil

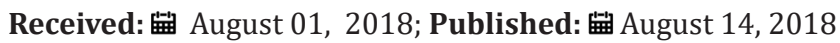 \\ *Corresponding author: Alexandre Cestari, Federal Institute of Education, Science and Technology of São Paulo, IFSP Campus Matão, \\ Brazil
}

\begin{abstract}
The biocompatibility of calcium phosphates is well known in the scientific community and they can be used as a replacing or filling material in cases that the bone was severely broken, demineralised by osteoporosis or bacterial activity and after removal of tumours. This work showed different synthesis, characterizations and applications of tricalcium phosphate (TCP) as a bone substitute. It was observed that the crystalline phase, synthesis method, porosity, grain size and biodegradability of TCP can improve the biocompatibility and osseointegration of biomaterials. In this review, TCP showed superior characteristics than hydroxyapatite. In conclusion, TCP is a promising material and further researches are needed to enhance the production and use as a bone substitute.
\end{abstract}

Keywords: Tricalcium Phosphate; TCP; Bone Substitute; Biomaterial; Hydroxyapatite

Abbreviations: TCP: Tricalcium Phosphate; HAP: Hydroxyapatite; XRD: X-Ray Diffraction; FTIR: Fourier Transform Infrared Spectroscopy; ASAP: Accelerated Surface Area and Porosimetry; SEM: Scanning Electron Microscopy

\section{Introduction}

The biocompatibility of calcium phosphates is well known in the scientific community and it is related to the bone and teeth compositions that contain the same elements and similar crystalline and porous structures of these materials. The phosphates can be used as a replacing or filling material in cases that the bone was severely broken or demineralised by osteoporosis or bacterial activity and to repair bones after removal of tumours [1,2]. There are compositions and crystalline structures with different physical and chemical properties. In example, the most studied materials are anhydrous or di hydrated calcium phosphate, octa calcium phosphate, hydroxyapatite (HAp), fluoroapatite and alpha or beta tricalcium phosphate (TCP). Different materials show unique behaviour and in vivo or in vitro experiments are necessary to evaluate the biocompatibility, dissolution, osteo inductive and osteoconductive properties. In Biomedicine, the use of TCP as coatings on biodegradable metallic alloys for orthopaedic devices is an alternative to improve osseointegration and to avoid faster corrosion of the implants [3-5]. HAp is widely applied in clinical proceedings and, nowadays, TCP is being studied as a bone substitute, with superior activity. The aim of this work was to elucidate TCP synthesis, characterizations and applications.

\section{Materials and Methods}

\section{Porous Tricalcium Phosphate}

Kalkura et al. synthesized by hydrothermal route a new porous calcium phosphate composed of non-resorbable HAp with resorbable alpha and beta-TCP and evaluated the material in simulated body fluid (SBF) [6]. The blend of TCP with HAp was made because porous HAp promotes osteo conductivity, but the non resorbable characteristic can decrease the rate of integration of the new bone structure. However, the soluble phase of TCP can induce osteogenesis and accelerate bone formation by the release of ions that act as a seed for new bone formation $[7,8]$. The material was characterized by X-Ray Diffraction (XRD) to evaluate the formation of crystalline phases, Fourier Transform Infrared Spectroscopy (FTIR) and Raman Spectroscopy to identify the chemical bonds, Accelerated Surface Area and Porosimetry (ASAP) to determinate surface area and pore size, Scanning Electron Microscopy (SEM) to visualise the porous network and apatite layer formation after SBF treatment. The authors concluded that the sample showed enhanced biocompatibility and excellent bioactivity for biomedical applications [6].

\section{Tricalcium Phosphate Scaffolds}

Le et al. developed a new class of beta-TCP scaffolds with a grain size of $200 \mathrm{~nm}$ through a two-step chemical precipitation and porogen burnout techniques. They evaluated the degradation by in vitro tests and characterized the material by XRD, SEM, compressive strength and porosity by the Archimedes method. [9]

Porous materials with diameters between 50 and $150 \mu \mathrm{m}$ can stimulate osteoid formation, and between 150 and $500 \mu \mathrm{m}$ can lead to the formation of mineralized bone directly, with osteo 
conduction. According to the results, the scaffolds showed porosity between 50 and $65 \%$, with pores ranging from 100 to $300 \mu \mathrm{m}$, with improved compressive strength and showed great potential for application in bone reconstruction. [9-11

\section{HAp and TCP: in vivo evaluation}

Hardouin et al. evaluated HAp and beta-TCP by in vivo tests. They implanted the materials in the middle shaft of rabbit femurs for 24 weeks and analyzed the biocompatibility and bioactivity. After 14 days, the TCP showed more osteoblasts colonizing the contact surface than the HAp. After 28 days, osteoblasts went through the porous structure of TCP and after eight weeks, new bone growth towards the centre of the implant. In the HAp group, bone in growth remained only at the peripheral region[12]. After 12 and 24 weeks, it was observed good bone penetration in the whole implant, with TCP biodegradation. The results showed that TCP increased the calcification rate and the bone ingrowth, compared to HAp [12].

\section{Discussion}

This work showed different synthesis, characterizations and applications of TCP as a bone substitute. It was observed that the crystalline phase, synthesis method, porosity, grain size and biodegradability of TCP can improve the biocompatibility and osseointegration of biomaterials. In this review, TCP showed superior characteristics than HAp. In conclusion, TCP is a promising material and further researches are needed to enhance the production and use as a bone substitute.

\section{Acknowledgment}

To Federal Institute of Education, Science and Technology of São Paulo - IFSP - Campus Matão.

\section{References}

1. Ogose A, Hotta T, Kawashima H, Kondo N, Gu W, et al. (2005) Comparison of Hydroxyapatite and Beta Tricalcium Phosphate as Bone Substitutes
After Excision of Bone Tumors. J Biomed Mater Res Part B: Appl Biomater 72(1): 94-101.

2. Hirata M, Murata H, Takeshita H, Sakabe T, Tsuji Y, et al. (2006) Use of purified beta-tricalcium phosphate for filling defects after curettage of benign bone tumours. International Orthopaedics (SICOT) 30(6): 510513.

3. Shadanbaz S, Dias GJ (2012) Calcium phosphate coatings on magnesium alloys for biomedical applications: A review. Acta Biomaterialia 8(1): 2030.

4. Dorozhkin SV, Epple M (2002) Biological and Medical Significance of Calcium Phosphates. Angew. Chem. Int 41(17): 3130-3146.

5. Carrodeguas RG, Aza AH, Turrillas X, Pena P, Aza S, et al. (2008) New Approach to the beta to alpha Polymorphic Transformation in Magnesium-Substituted Tricalcium Phosphate and its Practical Implications. J Am Ceram Soc 91(4): 1281-1286.

6. Vani R, Girija EK, Elayaraja K, Parthiban SP, Kesavamoorthy R, et al. (2009) Hydrothermal synthesis of porous triphasic hydroxyapatite/ alpha and beta tricalcium phosphate. J Mater Sci: Mater Med 20: S43-S48.

7. Daculsi G (1998) Biphasic calcium phosphate concept applied to artificial bone, implant coating and injectable bone substitute. Biomaterials 19(16): 1473-1478.

8. Castilho M, Moseke C, Ewald A, Gbureck U, Groll U, et al. (2014) Direct 3D powder printing of biphasic calcium phosphate scaffolds for substitution of complex bone defects. Biofabrication 6: 015006-015018.

9. Zhang F, Chang J, Lin K, Lu J (2008) Preparation, mechanical properties and in vitro degradability of wollastonite/tricalcium phosphate macroporous scaffolds from nanocomposite powders. J Mater Sci: Mater Med 19: 167-173.

10. Lin KL, Chang J, Zeng Y, Qian WJ (2004) Preparation of macroporous calcium silicate ceramics. Mater Lett 58: 2109-2113.

11. Liu DM (1997) Influence of Porosity and Pore Size on the Compressive Strength of Porous Hydroxyapatite Ceramic. Ceram Int 23: 135-141.

12. Lu JX, Flautre B, Anselme K, Hardouin P (1999) Role of interconnections in porous bioceramics on bone recolonization in vitro and in vivo. J of Mat Science: Mat In Medicine 10:111-120.

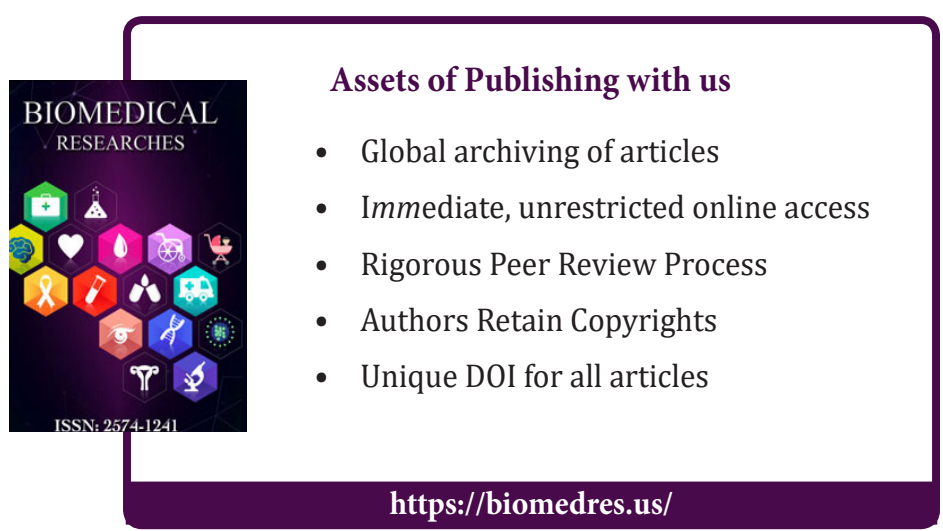

https://biomedres.us/
ISSN: 2574-1241

DOI: 10.26717/BJSTR.2018.08.001580

Alexandre Cestari. Biomed J Sci \& Tech Res



This work is licensed under Creative Commons Attribution 4.0 License

Submission Link: https://biomedres.us/submit-manuscript.php 